\title{
Long-term monitoring and assessment of a precast continuous viaduct
}

\author{
Helder Sousa, Carlos Sousa, Afonso S. Neves, João Bento, Joaquim Figueiras
}

http://www.tandfonline.com/doi/abs/10.1080/15732479.2011.614260?tab=permissions\#tabModule

Article published online: 19 SEP 2011

DOI: $10.1080 / 15732479.2011 .614260$

Copyright $\odot 2013$ Taylor \& Francis

Structure and Infrastructure Engineering

Volume 52, pages 777-793, August 2013 


\title{
Long-term monitoring and assessment of a precast continuous viaduct
}

\author{
Helder Sousa ${ }^{\mathrm{a},{ }^{,},}$, Carlos Sousa ${ }^{\mathrm{a}}$, Afonso S. Neves ${ }^{\mathrm{a}}$, João Bento ${ }^{\mathrm{b}}$, Joaquim \\ Figueiras $^{\mathrm{a}}$
}

\author{
${ }^{a}$ LABEST, Faculty of Engineering, University Porto \\ ${ }^{b}$ BRISA - Auto-Estradas de Portugal S. A.
}

Corresponding author: Helder Sousa; LABEST, Faculty of Engineering, University Porto, Rua Dr. Roberto Frias s/n, 4200-465 Porto, Portugal; Tel.: +351225081823; fax: +351225081835; e-mail: mail@hfmsousa.com

\begin{abstract}
Precast girders have recently been widely employed in the construction of bridges and viaducts. The new bridge over the Tagus River in Portugal, the Lezíria Bridge, comprehends a $9160 \mathrm{~m}$ long South Approach Viaduct, which was built with precast girders made continuous in situ. Given the relevance of this construction, a long-term monitoring system was implemented and measurements were taken since the start of the construction. The observed parameters were concrete strains and temperatures, deck rotations, joint displacements, accelerations and also environment temperature and relative humidity. The work presents the precast structure, the monitoring system, and the appraisal of a statistical procedure for the long-term assessment of the structural behaviour. This procedure is based on prediction models, which establish the normal correlation patterns between environmental and material parameters (such as concrete temperature and shrinkage strains), and the observed structural response in terms of strains, rotations and movements of expansion joints. The calculation of the normal correlation pattern comprehends the minimization of a square error. By applying the prediction model to the structural response measured in the South Approach Viaduct of the Lezíria Bridge, it was found that this methodology is a feasible tool for real-time damage detection of bridges.
\end{abstract}

Keywords: Precast concrete bridges, long-term monitoring, numerical validation prediction models, correlation patterns.

\section{Introduction}

Structural monitoring is an issue that receives more and more research interest and Bridge Health Monitoring Systems (BHMS) have been a subject of increasing international relevance. While in the past attention was focused on sensors, the emphasis is shifting to the practical implications regarding the acquisition, collecting and processing of data (Van der Auweraer and Peeters 2003). Today it is possible to continuously and remotely monitor highly instrumented structures, with a high degree of automation. Present solutions are versatile enough to allow for surveillance tasks to 
be remotely carried out in a cost effective manner (Bergmeister and Santa 2001; Chang, et al. 2009).

The condition assessment of a given structure may be performed by comparing monitoring results against numerical models that describe the predicted structural behavior. Finite Element models (FEM) have been widely employed in those calculations. FEM make it possible to calculate the long-term behavior of bridge structures, considering the influence of concrete creep and shrinkage, temperature and imposed loads. Accurate estimates of the actual structural behavior can only be obtained if the relevant concrete properties (creep, shrinkage and modulus of elasticity) are determined through adequate tests and the real temperature history is known. However, this information is not always available. Moreover, the detailed analysis through FEM, considering the real sequence of construction, is a time consuming process.

An alternative approach for condition assessment consists in using the data collected by the monitoring systems to establish the statistical models which describe the normal correlation pattern between non-structural parameters (such as temperature and shrinkage strains) and the observed structural response (strains, rotations and movements of expansion joints). Some authors have already followed this path. Indeed, the long-term monitoring of the Cogan and Grangetown viaducts (UK) were studied by Howells et al. (Howells, et al. 2005) and Barr et al. (Barr, et al. 1997). These authors found that a linear relationship could be established between the observed strains and temperatures, although these temperatures differ slightly, depending on the season and the segment location within the span. Ni et al. (Ni, et al. 2007) have also analyzed the relationship between observed temperatures and bridge response. However, unlike the former, whose study was based on environmental temperatures, Ni et al. used temperatures measured inside the bridge cross sections. This enabled the authors to 
establish a normal correlation pattern between temperatures and structural response. The correlation model was used to establish alarms to detect future monitoring data that disobey the normal pattern. In this authors' study, the analysis of the structural response was focused on the movement of the expansion joints, envisioning the scheduled interval for replacement of expansion joints.

The long-term behavior of structures such as the South Approach Viaduct of the Lezíria Bridge, in Portugal, is complex. In fact, it is well known that significant stress redistributions occur over the service life, due to concrete creep and shrinkage deformations and the evolution of the structural system during the construction phases. Therefore, a monitoring system was implemented to follow the structural behavior since the beginning of the construction.

In this paper, the above mentioned structure is briefly described and the implemented monitoring system is presented. Then, the main steps involved in the treatment of the experimental data are exposed.

At that stage, the comparison between the experimental results and the outcome of FE models is addressed. It was concluded that FE models are able to provide approximate estimates of the long-term behavior if detailed information about the material properties and the construction procedure are available. However, the analysis procedure is time consuming and the FE results hardly match the observed values with a high degree of accuracy, because the definition of the material properties, the environmental parameters, the structure geometric characteristics and the applied loads always involve some degree of approximation.

Therefore, a real time assessment procedure was developed to calculate the expected value of the monitored parameters at a given time. The developed approach is based on prediction models, which establish the normal correlation patterns between 
non-structural parameters and the observed structural response. That correlation pattern is established in the first years after construction, assuming that the structure has a healthy behavior in that period. For later ages, the expected values can then be compared with the observed ones, so that anomalies can be detected if the difference exceeds a given threshold limit. One of the strengths of this methodology lies in the fact that it provides quick calculation results with minimum time and computational efforts. Moreover, it is suitable for implementation in automatic monitoring systems, which trigger an alarm if unexpected values occur. In this context, this work aims at contributing to the development of systems devoted to the management and maintenance of bridges.

\section{The precast viaduct}

The Lezíria Bridge, a, 11,670m long structure, is composed of three distinct substructures: (i) the north approach viaduct; (ii) the main bridge crossing the Tagus River; (iii) and the longest one, the south approach viaduct, with a total length of $9160 \mathrm{~m}$. The south approach viaduct is a partially precast structure and is composed of 22 elementary viaducts, whose total length (i.e., distance between expansion joints) ranges from $250 \mathrm{~m}$ to $530 \mathrm{~m}$. The most common span length is $36 \mathrm{~m}$.

Each elementary viaduct is composed of a continuous deck monolithically connected to the piers. The foundation is provided by piles, which have the same cross section as the piers, thus forming a pier-pile element. Transition piers were adopted to establish the connection between the different elementary viaducts. In the transition, one of the elementary viaducts is connected to the transition pier by means of fixed pot bearings whereas the other is supported by sliding guided bearings.

The deck slab, which is $29.95 \mathrm{~m}$ wide, is made up of four $1.75 \mathrm{~m}$ high, precast, $\mathrm{U}$ shaped girders and a $0.25 \mathrm{~m}$ thick slab. The girders are prestressed by means of 
pretension strands and were made in a factory specifically build at the construction site. The prestress release was carried out approximately 12 hours after casting.

The slab is composed of precast planks and a cast-in-place layer. The monolithic connection between spans is established through a cast-in-place diaphragm and continuity reinforcement. Moreover, in the region above the piers, the deck slab is prestressed by straight post-tensioning cables. The continuity diaphragm is also monolithically connected to the piers, which have a circular cross section and a diameter of $1.5 \mathrm{~m}$. On the top of the pier, there is an octagonal capital whose maximum dimension reaches $1.7 \mathrm{~m}$, so that the girders can be positioned without conflicts with the pier reinforcement. In the transverse direction, a precast beam connects the capitals of each two pillars. The piles cross alluviums with variable constitution, and the maximum deepness reached by each pile varies between $35 \mathrm{~m}$ and $60 \mathrm{~m}$. The concrete piles were cast inside steel tubular elements, which were installed by using a vibratory pile hammer (COBA-PC\&A-CIVILSER-ARCADIS 2006).

Fig. 1 depicts an image of the viaduct construction and a detail of the continuity diaphragm during construction.

\section{The monitoring system}

The decision to monitor this structure was motivated by two main reasons. Firstly, it is a very long structure, made with a repetitive precast solution; consequently, the actual performance of the structural solution can be observed by monitoring part of the structure; in this way, the bridge owner can be aware of the long-term structure performance, spending a reduced value with respect to the overall structure cost. Secondly, important stress redistributions occur in these structures, due to the employed construction sequence and the concrete creep and shrinkage properties. Therefore, the 
monitoring results of this complex behavior are expected to provide valuable data for the validation of a proper numerical model.

Two out of the 22 elementary viaducts were monitored: the V1S and the V14S (Fig. 2). Before the start of the construction, a monitoring plan was developed, which indicates the parameters of interest and the critical cross sections that were selected for monitoring (Figueiras, et al. 2007). These viaducts were extensively instrumented, namely with vibrating wire strain gauges to measure concrete deformations (CD), thermistors and resistive temperature detectors to measure concrete temperature (CT), inclinometers to measure girder rotations about the horizontal axis of the deck cross section $(\mathrm{RO})$, linear variable differential transformers to measure the longitudinal relative displacement between the deck and the supports at the sliding guided bearings (BD) as well as temperature and humidity sensors to measure environmental conditions (TE, RH). Fig. 2 presents the elevation of the monitored viaducts, with indication of the cross sections in which embedded sensors were installed, as well the external sensors location, while Fig. 3 illustrates the distribution of the embedded sensors in two typical cross sections: mid-span and support cross sections. Some of the embedded sensors are labeled in Fig. 3 for future reference in the paper.

Eight concrete prisms, with dimensions $15 \mathrm{~cm} \cdot 15 \mathrm{~cm} \cdot 55 \mathrm{~cm}$, were cast for measurement of shrinkage deformations. These prisms were instrumented with strain gauges and temperature sensors and subjected to the same curing conditions of the monitored structural elements, to achieve as a realistic representation as possible, of the deck concrete shrinkage. Four prisms were made with girder concrete and subjected to the curing procedure applied to the precast beams; the other ones were made with slab concrete (from the mid-span region of the second span). For each concrete composition, half of the prisms were subjected to the surrounding environmental conditions (although 
sheltered from rain), whereas the others were kept in the interior of the box girder since the pouring of the second-span slab of each viaduct.

Given the aggressiveness of the concreting operations, the embedded sensors (vibrating wire strain gauges and temperature sensors) were fixed to the reinforcement, near the concrete surface, by using square steel bars, which allowed a robust installation (Fig. 4-a). In general, measurements started before the concreting operations.

Consequently, it was necessary to install cables through provisory and safe paths and acquisition systems in adequate protection boxes, to prevent potential damage due to the construction works. The available time for these works was always short. For that reason, the installation of all the embedded sensors was a demanding task. The bridge deck instrumentation comprised two main phases: (i) the instrumentation of the precast girders, which started in the precast plant; (ii) the instrumentation of the deck slab, which started before the in situ concreting.

The external sensors were installed after the structure was cast. Inclinometers with great inertial sensitivity were employed to measure the deck rotation about the horizontal axis. These sensors were rigidly attached to a metallic base, which was previously fixed to the precast girder surface and properly leveled (Fig. 4-b). The metallic base is provided with a mechanism allowing the leveling of the sensor without removing it. Both temperature and humidity sensors were installed in two different environments: (i) inside the box girders; (ii) outside the precast girders, sheltered from rain. Given that it is not possible to access the interior of the box girders after the end of the construction, internal sensors were fixed to a movable sealed device, so that the sensors could be accessed in case of malfunctioning or for maintenance operations. Linear Variable Differential Transformers (LVDTs) were installed to monitor the bearing displacements of the viaducts. These sensors were installed after the expansion 
joint was placed. The installation was made by fixing one sensor end to the pier top, and the other to the lateral face of the girder that is supported by sliding guided bearings. In order to prevent damage and to guarantee the appropriate mechanical protection, all the external sensors were encapsulated in a protection box (ventilated in the case of the environmental parameters measuring). In the case of the LVDT, the mechanical protection was performed by a stainless steel shelter (Fig 4-c).

\section{Interpretation of concrete strains}

A large number of vibrating wire strain gauges were employed to evaluate concrete strains. The results obtained with these sensors must be carefully interpreted. According to Eq. (1), the strain reading, obtained by direct correlation with the wire vibration frequency, may be decomposed into three basic components: (i) the instantaneous deformations that occur at specific instants of time, $\mathrm{t}_{0}$, caused by events such as loadings or prestressing operations, $\varepsilon_{\mathrm{ci}}(\mathrm{t} 0)$; (ii) the delayed deformation due to long term effects such as creep and shrinkage, $\varepsilon_{\mathrm{cs}+\mathrm{cc}}(\mathrm{t})$; the deformation due to temperature variations, $\varepsilon_{\mathrm{cT}}(\mathrm{t} 0)$.

$$
\varepsilon_{c}(t)=\sum_{i} \varepsilon_{c i}\left(t_{0}\right)+\varepsilon_{c s+c c}(t)+\varepsilon_{c T}(t)
$$

\subsection{Instantaneous deformations}

If the cause of a given instantaneous deformation, $\varepsilon_{\mathrm{ci}}\left(\mathrm{t}_{0}\right)$, is not known, then it is not possible to interpret correctly the corresponding results. This is even more complicated in a structure that was built across different construction phases. For this reason, information about all the relevant construction events was recorded and compiled in the quality control report (TACE 2007). This document allows the identification of the construction event that matches a given instantaneous deformation. 


\subsection{Time-dependent deformations}

The time-dependent deformations are mainly due to the concrete creep and shrinkage.

The relaxation of prestressing steel is also responsible for long-term deformations, however both the magnitude and the variability of this phenomena are significantly less important than those due to creep and shrinkage. In this context, shrinkage was measure in eight prisms, as mentioned before.

Figs. 5 and 6 depict the monitored strains in the shrinkage prisms of the V14S viaduct without the temperature effect (analogous results were obtained in the other monitored viaduct). In these two figures, the shrinkage estimated according to the Eurocode 2 (European Committee 2004) is also shown, and it was based on the concrete compressive strength measured in cubes (TACE 2007), considering a notional crosssection size of $150 \mathrm{~mm}$, and taking into account the fact that the concrete is made of high strength rapid hardening cements. Two different values were considered for the relative humidity $(\mathrm{RH}=70 \%$ and $\mathrm{RH}=90 \%$ ), which correspond to the average observed values in the exterior and interior environments. These figures show that the shrinkage prisms provide relevant information to support the interpretation of the monitoring results, as the code estimates do not precisely follow the observed values:

- even though the Eurocode 2 estimates might yield similar results for both concretes, there are significant differences between the actual shrinkage development in both materials, due to their different compositions and curing conditions;

- the girder concrete exhibits high shrinkage values in the first days after casting, which is not observed in the slab concrete, for which the Eurocode 2 overestimates the deformations;

- the observed shrinkage curves exhibit a seasonal variation, which has already been detected by other authors (Santos 2002; Santos 2007); shrinkage increases in the warm and dry months and decreases in the winter, but, again, this effect is not taken into account by the code estimates.

\subsection{Temperature effect on deformations}


Sensors used to measure concrete deformations are influenced, in general, by concrete temperature variation. Vibrating wire strain gauges are sensitive to both the wire and the concrete temperatures. Therefore, these sensors are usually provided with an internal thermistor, so that the temperature influence can be taken into account. The concrete strain can thus be obtained through the following equation:

$$
\varepsilon_{c}(t)=G F \cdot\left(f_{2}^{2}-f_{1}^{2}\right)+k \cdot \Delta T
$$

where $G F$ is the sensor gauge factor, $f_{1}$ and $f_{2}$ represent the initial and the actual (at the time $t$ ) vibrating wire frequency respectively and $\Delta T$ is the temperature variation. If the total concrete deformation is to be obtained, then only the wire temperaturedependent deformation must be compensated. In this case, the parameter $k$ takes the value:

$$
k=\alpha_{\text {wire }}
$$

where $\alpha_{\text {wire }}$ represents thermal dilation coefficient of the wire. The manufacturer usually gives this value and it takes the value $11 \mu \varepsilon /{ }^{\circ} \mathrm{C}$ for the sensors used in this work (Gage Technique International). However, it is often more convenient to obtain a strain reading which does not include the free thermal deformation. This can be achieved by taking the following parameter $k$ :

$$
k=\left(\alpha_{\text {wire }}-\alpha_{c}\right)
$$

where $\alpha_{c}$ represents the thermal dilation coefficient of the concrete. If the temperature compensation is carried out in this way, then the obtained strain $\varepsilon_{c}(t)$ only includes stress dependent deformations (i.e., deformations caused by concrete stresses) and shrinkage strains. The strain obtained through this compensation procedure is suitable for comparison with the results of numerical models.

The accurate evaluation of the temperature effect depends on the correct estimate of the parameter $\alpha_{c}$. For this reason, a set of prisms (made with the same 
concrete that was used in the structure and instrumented with the same type of transducer) was tested in a climatic chamber. The test procedure consisted in applying different temperature cycles. The temperature variation was slow, so that a uniform temperature distribution could be obtained in the prisms. The temperature ranged from 5 ${ }^{\circ} \mathrm{C}$ to $40{ }^{\circ} \mathrm{C}$, which broadly corresponds to the range of temperatures observed in the structure. The results obtained for the parameter $k=\left(\alpha_{\text {wire }}-\alpha_{c}\right)$ that eliminates the sensitivity to the free thermal deformation are characterized by an average value $\mu=$ $3.1 \mu \varepsilon /{ }^{\circ} \mathrm{C}$ and a standard deviation $\sigma^{2}=0.09 \mu \varepsilon^{2} /{ }^{\circ} \mathrm{C}^{2}$ (Sousa and Figueiras 2009). Fig. 7 presents the results obtained in one typical concrete prism. The evolution of both the concrete temperature and the climatic chamber temperature is also represented in this figure. These results reveal that the thermal dilation coefficient of the employed concrete is approximately equal to $7.9 \mu \varepsilon /{ }^{\circ} \mathrm{C}$. This value is similar to that obtained by other authors (Santos 2007; Kada, et al. 2002).

\section{Comparison between monitoring and FEM results}

A finite element analysis of the V14S viaduct was carried out, aiming at evaluating whether a detailed numerical model could provide similar results to the ones that were experimentally obtained. A good agreement between field measurements and FEM results validates the monitoring procedures and encourages the effective use of this information in surveillance and assessment tasks. Two different analyses were performed: (i) a time-dependent phased analysis of the viaduct response, comprising both construction and service phases; (ii) a linear-elastic analysis of the structural behavior during the proof load test.

The time-dependent analysis was carried out through a FEM with beam elements. A phased analysis was performed, in which the actual construction sequence was modeled, according to the recorded sequence of construction events (TACE 2007). 
In this analysis, the constitutive model adopted to describe the concrete behavior allows for the consideration of the creep, shrinkage and cracking effects. The definition of the material properties was also based on the information collected during the qualitycontrol procedures (TACE 2007). Furthermore, the shrinkage variation was described by curves that were determined through a curve fitting procedure based on the results of the shrinkage prisms. Given that no creep measurements were available, the strain results observed in one of the precast girders before its erection was used to obtain additional information regarding the long-term behavior of the girder concrete. This was carried out by means of a retro-analysis procedure.

A 3D FEM with brick elements was used to analyze the local behavior of the connection between the deck and the piers during the proof load test. This is a zone of strong geometric discontinuity. Consequently, the strains that were monitored in this region (see Figs. 2 and 3) cannot be directly compared with results calculated by means of finite element beam models. Furthermore, modeling the whole viaduct with 3D brick elements would give rise to a complex and large model. Consequently, the following analysis strategy was adopted. The global analysis of the different load cases was carried out by a FEM with beam elements. Then, the local 3D model was used to determine the strains in the sensor locations when the corresponding cross section is subjected to a given bending moment. Note that the local 3D model comprises half a span, so that the boundary conditions can be properly simulated.

This paper presents some relevant and illustrative results. A comprehensive description of the employed numerical models and the obtained results can be found elsewhere (Sousa, et al. 2009; Sousa, et al. 2011).

\subsection{Short-term analysis (proof load test)}


Four different load positions were considered in the proof load test to maximize the stresses in each monitored cross section.

Fig. 8 depicts the results of the proof load testing for the 12 sensors that are located at upper level in the 4 monitored support cross sections. The calculated results are also plotted in this figure (note that the dashed lines do not correspond to the exact variation of strain between points; they are only represented to clarify the interpretation of the results). From the analysis of this figure, one may conclude that the strain distribution through the deck slab is not uniform. This is a consequence of both the geometric discontinuity due to the support diaphragm and the shear lag effects, which lead to lower strains in the transducer located on the symmetry axis. In Fig. 8, this effect is evident in both the experimental and the numerical results. Moreover, there is a remarkable agreement between the calculated and the observed results and a good repeatability of measurements in corresponding locations.

\subsection{Long-term analysis}

Fig. 9 presents the strain results for one of the monitored mid-span cross sections, during the construction and the first years in service. Some relevant construction stages are identified in this figure and the observed values (at 6 a.m.) are represented by symbols that identify the location of each point. Note that measurements started before the precast girder was cast and the strain values do not include the free thermal deformation.

This figure also depicts the results of two FEM analyses: (i) continuous lines represent calculations in which the concrete properties were based on monitored parameters (results of concrete cube tests, shrinkage measurements and retro-analyses for identification of the creep properties) (Sousa, et al. 2011), and (ii) dashed lines present the outcome of calculations in which the concrete properties are based on the 
Eurocode 2 estimates and the design specifications. Figs. 5 and 6 had already shown that the measured shrinkage deformations were significantly different from the code estimations. Therefore, it is not surprising that calculations based on estimated concrete properties lead to results significantly different from the observed ones. Differences are particularly important in the first weeks after casting the precast girder and before casting the continuity connection, because the deviation between the actual creep and shrinkage deformations and the estimated values are significantly higher in this period. In the long term, the agreement between measurements and calculations is improved, namely in the case of the girder strains. In the case of the deck slab, important differences exist even in the long term, because the actual slab shrinkage strains are lower than the estimated values (see Fig. 5).

Conversely, the outcome of the detailed analysis with concrete properties based on measured parameters closely agrees with the observed strains. Therefore, important conclusion could be taken from the calibrated FEM analyses: they revealed that the actual structure behavior is in accordance with the expected response, and the good agreement and correlation between the different measured parameters gives confidence in the monitoring system. FEM analyses proved to be relevant for validation of the measured values. However, detailed analyses are time-consuming and a lot of detailed information is necessary for the accurate calculation of the long-term structure response. Thus, the use of simpler approaches for real-time long-term assessment of the structure performance is encouraged and justified.

\section{Prediction models}

\subsection{Model description}

A possible approach for real time assessment of the structure condition was evaluated within the scope of the present work. This procedure consists in obtaining the normal 
correlation pattern between the structural response (strains, rotations and movements of expansion joints) and basic information provided by environmental and material parameters (such as concrete temperature and concrete shrinkage measurements). If the new structure has a healthy behavior in the first years after construction, it is possible to determine the normal correlation pattern between different measurements. These correlations can be regarded as prediction models, which can be used to assess the adequacy of the structure behavior in the future.

The proposed model is described by Eq. (5), where the model prediction $y_{\text {model }}(t)$ for a given parameter (strains, rotations or movements of expansion joints) at a given time $t$ is estimated from a linear combination of measurements $x_{\text {measure }, i}(t)$ weighted by $w_{i}$. The predictor parameters $x_{\text {measure }, i}(t)$ must comply with the following conditions: (i) the structure response must be sensitive to the value of this parameters; (ii) they must not depend on the soundness of the structure behavior, i.e., they must represent material properties or environmental characteristics; (iii) minimal correlation should exist between the predictor parameters. It is desirable that these parameters correlate highly with the dependent ones, but the existence of high correlation between the predictors (multicollinearity) leads to imprecise determination of the coefficients $w_{i}$, inaccurate estimates and inexact tests on the regression coefficients (Montgomery and Runger 2003). In this work, the predictor parameters consist of concrete temperature and concrete shrinkage measurements, for the reason that these variables strongly affect the time variation of the structure response.

$$
\begin{gathered}
y_{\text {model }}(t)=\sum_{i=1}^{k} w_{i} \cdot x_{\text {measure }, i}(t) \\
y_{\text {measure }}(t)=y_{\text {model }}(t)+\varepsilon
\end{gathered}
$$


The problem unknowns consist of the set of weights $w_{i}$. The problem is solved by calculating the value of the weights $w_{i}$ which minimize the differences, $\varepsilon$, between the actual measurement $y_{\text {measure }}(t)$ and the model prediction, $y_{\text {model }}(t)$. The optimal solution is the one that minimizes the function $R$, which represents the sum of the squared errors, as represented by Eq. (6). The minimum of the function $\mathrm{R}$ is found by setting the gradient to zero. This problem has an unique solution because $R$ is a quadratic function, $y_{\text {model }}(t)$ being a linear combination of a set of measurements.

$$
\begin{aligned}
& R=\sum_{i=1}^{n}\left(y_{\text {measure }}\left(t_{i}\right)-y_{\text {mod } e l}\left(t_{i}\right)\right)^{2}=\sum_{i=1}^{n} \varepsilon_{i}^{2} \\
& \left.R_{\min } \Rightarrow \frac{\partial\left(\sum_{i=1}^{n} \varepsilon_{i}^{2}\right)}{\partial w_{j}}\right|_{w_{0}, w_{1}, \ldots, w_{k}}=0 \quad j=1,2, \ldots, k
\end{aligned}
$$

The observation period (i.e., the set of times $t_{i}$ ) over which the minimization problem is formulated must also be established. In this type of problems, it is common to use a fraction of the total available period of observation, a so called "training window". The appropriate size of the training window can be determined by making several calculations with increasingly larger dimensions. The adequate time window is the one that lead to the same set of weights $w_{i}$ that would be obtained if larger training windows were considered.

It is worthy of note that the size of the training window depends on the phenomena under analysis. If the prediction model is devised for long-term structural analyses, then a minimum size of one year might be necessary, for the reason that the concrete temperatures and delayed deformations have a seasonal variation with a yearly period.

Once the set of weights is determined, the model may be used to calculate the expected structure response $y_{\text {model }}(t)$ for the remaining time window. If the structure has 
a normal behavior, the magnitude of the error $\varepsilon$ shows the robustness of the prediction model.

This algorithm was implemented in an existing software devoted to structural monitoring - MENSUSMONITOR. Its user-friendly interface and capacity to connect to MySQL databases enabled efficient data handling and simple calculation procedures. More details about this tool can be found elsewhere (Sousa, et al. 2008).

\subsection{Application to the precast viaduct}

\subsubsection{Problem description}

The prediction model was used to analyze the outcome of 24 transducers (12 from viaduct V1S and 12 from V14S): $4(2+2)$ bearing displacements $B D ; 4(2+2)$ deck rotations $R O$; and $16(8+8)$ concrete deformations $C D$, of four mid-span cross sections (Figs. 2 and 3). The predictor parameters $x_{\text {measure }, i}(t)$ were shrinkage deformations $S D$ observed in concrete prisms and concrete temperatures $C T$. Fig. 10 shows the location of the temperature sensors, which are positioned in three mid-span cross sections.

Tables 1 and 2 present the correlation coefficients $\mathrm{R}^{2}$ for all the measurements that are candidates to be used as predictor parameters, for viaducts V1S and V14S respectively. These tables show that high correlation exists within the subsets of temperatures and shrinkage measurements, with $\mathrm{R}^{2}$ always higher than 0.95 . This indicates a strong collinearity between measurements of each data set. Consequently, instability problems would occur in the calculation of the problem unknowns (the set of weights $w_{i}$ ) if more than 1 temperature and 1 shrinkage strain were taken as predictor parameters.

Therefore, only two independent variables were considered: the average of the shrinkage deformations $\mathrm{SD}_{\mathrm{AVG}}$ and the average of the concrete temperatures $\mathrm{CT}_{\mathrm{AVG}}$. The correlation coefficients between these variables are -0.281 and -0.542 for viaducts 
V1S and V14S, respectively. These correlations lead to Variance Inflation Factors of 1.1 and 1.4, respectively, which are clearly lower than 10 (the threshold value for multicollinearity) and robust model predictions can thus be achieved (Montgomery and Runger 2003). In this way, the prediction model given by Eq. (5) is expressed by Eq. (7).

$$
y_{\text {mod } e l}(t)=w_{1} \cdot S D_{A V G}(t)+w_{2} \cdot C T_{A V G}(t)
$$

The uniform depth of this bridge deck and the reduced thickness of both the deck slab and the girders justify the adoption of such a simple description of the temperature influence as presented in Eq. (7). However, in structures composed by thicker elements higher temperature gradients occur, which requires a more detailed definition of the temperature influence.

For this study, the observation period ranges from 24-12-2007 to 05-10-2009, with one sample per day, at $6 \mathrm{~h}$ a.m.

\subsubsection{Results and discussion}

Figs. 11 to 13 present the model predictions for three measurements: a concrete deformation, a deck rotation and a bearing displacement. For each measurement, the first graph depicts the time variation of measured values and model predictions. The training window size was taken as one year, which corresponds to $60 \%$ of the complete time range. However, the influence of this size is discussed below. The time variation of the error $\varepsilon$, i.e., the difference between measurements and model predictions, is also depicted in the graph. A second graph for each measurement shows histograms of the calculated errors, for both the training window and the remaining time window. The Gaussian distribution that best fits these histograms is also plotted.

The quality of the predictions for the different measurements can be compared by using the relative error concept, which is defined as the ratio between the calculated 
error at a given time $t$ and the range of variation of measurements over the complete time window (see e.g. Fig. 11). It was observed that the smallest relative errors occur in the case of the bearing displacement. For this parameter, the maximum relative error is $4.6 \%$, and $95 \%$ of the calculated errors are lower than $3.2 \%$. Moreover, the error average takes the value $0.45 \mathrm{~mm}$, which corresponds to $0.5 \%$ of the signal amplitude. This small value reveals that the models prediction follows the actual measurements and the error has a random nature. The correlation coefficient between predictions and measurements equals 0.99 , which confirms a good correlation.

The poorest performance of the prediction model was observed in the deck rotation, for which the maximum relative error is $20.3 \%$. However, even in this case, the error mean is approximately equal to zero. As for the concrete deformation, the maximum error takes the value $10.8 \%$. It is not surprising that the best agreement between measurements and predictions occur in the case of the bearing displacement. This is a global measurement that is influenced by the deformation of a large portion of the structure. Therefore, a good correlation between this parameter and the average values of temperature and shrinkage measurements was expected. On the other hand, local measurements such as concrete deformations are very sensitive to the local concrete behavior, which does not correlate so well with the adopted average predictor parameters.

The occurrence of errors is also justified by the fact that the structure response is also sensitive to other variables that were not considered as predictor ones: traffic loading, wind, insolation, rainfall, among others with minor importance. This is why the prediction of the deck rotations was the poorest one: this transducer is the most sensitive to traffic loadings. However, the fact that the mean error is approximately equal to zero shows that the adopted predictor variables play a key role in the long-term structure 
response. If the long-term performance is to be evaluated, then it is logical to analyze the average error over a certain period, so that the relevance of random deviations due to the ignored effects is reduced. Tables 3 to 5 display the maximum relative error for weekly and monthly averages, showing that the error significantly decreases if averaged values are taken into account. The most significant reduction occurs for the deck rotation when the training window is equal to $60 \%$ of the observation window, a fact that is justified by the aforementioned reasons.

\subsubsection{Discussion of the training window size}

Tables 3 to 5 show the variation of the maximum relative error as the training window size increases (taking values of $30 \%, 45 \%$ and $60 \%$ of the observation time window), for the three transducers under analysis. Besides the maximum individual error and the weekly and monthly averages, these tables also present the maximum error for a probability of occurrence of $95 \%$.

In general, the maximum errors decrease as the training window size increases. In the case of the deck rotation, similar results are obtained for window percentages of $45 \%$ and $60 \%$. As regards the bearing displacements, the training window size seems to have a minor influence on the calculation results. The influence of the training window size can be more clearly understood by analyzing Fig. 14, which depict the evolution of the set of weights $w_{i}$ (the problem unknowns) when the training window size varies from $1 \%$ to $100 \%$ of the total period. The figure shows that a very small training window ( $10 \%$ of the observation time) guarantees a good estimate of the weights $w_{i}$ for prediction of bearing displacements. This conclusion conforms to the fact that these global displacements are well correlated with the predictor variables: averages of the measured concrete temperatures and shrinkage strains. On the other hand, large training windows are required for the prediction of local concrete deformations as well as for 
deck rotations, because of lower correlation with the predictor variables. An extensive study was also performed for the remaining sensors and similar conclusions were gathered.

\subsubsection{Example of anomaly detection}

The usefulness of the prediction model can be demonstrated by the following real example. Fig. 15 shows the time variation of measurements and model predictions for one of the monitored bearing displacements. The time variation of the calculated error (difference between measurements and predictions) shows an abrupt variation at a specific time (09-05-2008 6h a.m.), which indicates some anomaly. After searching for possible explanations for this occurrence, it was found that this instant of time corresponded to maintenance operations that forced a temporary removal of the LVDT. Upon removal and replacement of the transducer, the reference was lost, which justifies the detected deviation in the graphical representation of the error.

This maintenance operation was carried out without communication with the authors. It could only be detected after the prediction model has been applied to the results of this transducer. Therefore, the usefulness of the prediction model is not restricted to the detection of structure deficiencies; it also detects anomalies in the monitoring results.

It must be highlighted that, in this work, the occurrence of anomalies is associated to abnormal deviations between the measured structural response and the model estimation. The normal correlation pattern is established for a period of time in which healthy behavior is assumed and the threshold limit is derived from the statistical analysis of the deviations in that period of time. However, the definition of threshold limits could be complemented with additional information based on numerical modeling 
considering the influence of variables such as traffic, wind, insolation and rainfall. That would be a more complex approach that is out of the scope of the present work.

\subsubsection{Global analysis of measured results}

Given the large number of the installed transducers, a subset of 24 sensors was selected for presentation in this work, as previously mentioned. Fig. 16 shows the calculated maximum relative errors, for a training window of $60 \%$ of the total period of observation. The label of each concrete deformation, $\mathrm{CD}$, includes the cross section as indicated in Fig. 2, the sensor location within the cross section, as shown in Fig. 3, and the identification of the viaduct. The position of each rotation and displacement measurements is shown in Fig. 2.

These results conform to the ones discussed before. Predictions of bearing displacements (with maximum relative errors lower than $4 \%$ for a probability of occurrence of 95\%) are significantly better than the model estimations for concrete deformations and deck rotations. However, it is worthy to note that the relative errors are calculated with respect to the time variation in the observation period under analysis. If the total range of variation was considered, then significantly lower relative errors would be obtained. Fig. 9 shows that the maximum strain variation in the precast girder amounts to $\sim 850 \mu \varepsilon$. Therefore, the relative error with respect to the total range of variation is approximately ten times lower than the one with respect to the range of variation in the period of observation $(\sim 80 \mu \varepsilon$ for the transducer in Fig. 11).

\section{Conclusions}

The present work focus on the monitoring and assessment of the long-term structural behavior of continuous viaducts made with precast girders. A real structure, which was recently built in Portugal, has been monitored since the beginning of its erection. The 
monitoring system was carefully planned, installed and protected so that it could provide long-term reliable results. This paper describes the viaduct, presents the monitoring system, and discusses the procedure employed to assess the structure's behavior. This procedure is based on FEM calculations and regression models. Some relevant conclusions could be drawn:

(1) The measurement of the time variation of relevant concrete properties and the identification of the timing of the various construction events provided relevant information for the development of accurate FE analysis. This conclusion stems from the difference between the actual time variation of the concrete deformations and those resulting from the design code predictions and also from the fact that the construction sequence strongly affects the structural behavior.

(2) The good agreement between field measurements and FEM results validates the monitoring procedures and encourages the effective use of this information in surveillance and assessment tasks.

(3) The prediction model presented might be applied to establish normal correlation patterns between material and environmental parameters (such as temperature and shrinkage strains) and the observed structural response (strains, rotations and movements of expansion joints). The problem unknowns are determined by considering an initial time window in which healthy behavior is assumed. The model can then be employed to calculate the expected response of the different transducers, for each point in time. The existence of abnormal differences between the expected values and the actual measurements reveals changes in the structural behavior or deficiencies in the monitoring system.

(4) The choice of the predictor variables and the size of the time window used are of relevance and their calculations were presented and discussed.

(5) The use of prediction models involves minimal time and computational efforts, if the algorithm is implement in dedicated software, such as MENSUSMONITOR, with direct access to the measurements database.

(6) The usefulness of the prediction model was demonstrated through a real example in which a maintenance operation with implications in the monitoring results was detected.

(7) The best agreement between measurements and model predictions were obtained for transducers, which are not very sensitive to variables other than the employed independent (i.e., predictor) variables. In this case, the best results were obtained for the relative displacements at the expansion joints.

Acknowledgements

The authors acknowledge the support from the Portuguese Foundation for Science and Technology through the Research Project PTDC/ECM/68430/2006 and the PhD grants SFRH/BD/29125/2006 and SFRH/BD/25339/2005 attributed to the first and second authors. Support from the contractor consortium, TACE, and the infrastructure owner, BRISA, is also gratefully acknowledged.

\section{References}


Barr, B. I. G., J. L. Vitek, and M. A. Beygi. "Seasonal Shrinkage Variation in Bridge Segments." Materials and Structures 30, no. 196 (1997): 106-11.

Bergmeister, K., and U. Santa. "Global Monitoring Concepts for Bridges." Structural Concrete 2, no. 1 (2001): 29 -39.

Chang, Sung-Pil, Jaeyeol Yee, and Jungwhee Lee. "Necessity of the Bridge Health Monitoring System to Mitigate Natural and Man-Made Disasters." Structure and Infrastructure Engineering: Maintenance, Management, Life-Cycle Design and Performance 5, no. 3 (2009): 173 - 97.

COBA-PC\&A-CIVILSER-ARCADIS. "Construção Da Travessia Do Tejo No Carregado Sublanço A1/Benavente, Da A10 Auto-Estrada Bucelas/Carregado/Ic3 " In Empreitada de Concepção, Projecto e Construção da Travessia do Tejo no Carregado, 2006.

European Committee, Standardization. Eurocode 2 En 1991-1-1 Design of Concrete Structures Part 1-1 General Rules and Rules for Buildings. Vol. 2e ed. Brussels: CEN, 2004.

Figueiras, Joaquim, Carlos Félix, Helder Sousa, and Helena Figueiras. "Construção Da Travessia Do Tejo No Carregado Sublanço A1/Benavente, Da A10 AutoEstrada Bucelas/Carregado/Ic3: Projecto Executivo Monitorização Estrutural E De Durabilidade 0 - Apresentação." LABEST, Faculty of Engineering of the University of Porto, 2007.

Gage Technique International, Lda "The Effect of Temperature on Vibrating Wire Strain Gauges." United Kingdom: Gage Technique International, Lda.

Howells, R. W., R. J. Lark, and B. I. G. Barr. "A Study of the Influence of Environmental Effects on the Behaviour of a Pre-Stressed Concrete Viaduct." Structural Concrete 6, no. 3 (2005): 91-100.

Kada, H., M. Lachemi, N. Petrov, O. Bonneau, and P. Aïtcin. "Determination of the Coefficient of Thermal Expansion of High Performance Concrete from Initial Setting." Materials and Structures 35, no. 1 (2002): 35-41.

Montgomery, Douglas C., and George C. Runger. Applied Statistics and Probability for Engineers. 3rd ed. ed: John Wiley, 2003.

Ni, Y. Q., X. G. Hua, K. Y. Wong, and J. M. Ko. "Assessment of Bridge Expansion Joints Using Long-Term Displacement and Temperature Measurement." Journal of Performance of Constructed Facilities 21, no. 2 (2007): 143-51.

Santos, Luís Miguel Pina de Oliveira. Observação E Análise Do Comportamento Diferido De Pontes De Betão, Phd Thesis. Lisboa: LNEC, 2002.

Santos, Teresa Oliveira. Retracção Do Betão Em Pontes Observação E Análise. $1^{\text {a }}$ ed ed, Phd Thesis. Lisboa: LNEC, 2007.

Sousa, C. , H. Sousa, A.S. Neves, and J. Figueiras. "Numerical Evaluation of the Long-Term Behavior of Precast Continuous Bridge Decks." Journal of Bridge Engineering, 10.1061/(ASCE)BE.1943-5592.0000233 (Feb. 15, 2011) (2011).

Sousa, C., H. Sousa, A. Neves, and J. Figueiras. "Finite-Element Analysis of the LongTerm Behavior of the Precast Access Viaduct of the Leziria Bridge." LABEST, Faculty of Engineering of the University of Porto, 2009.

Sousa, H., A. Dimande, A. Henriques, and J. Figueiras. "Mensusmonitor - Tool for the Treatment and Interpretation of Experimental Results in Civil Engineering." Paper presented at the CCC 2008 - Challenges for Civil Construction, FEUP Faculty of Engineering, University Porto, Porto, 2008.

Sousa, H., and H. Figueiras. "Experimental Evaluation of Thermal Compensation for Vibrating Wire Strain Gauges Placed in Concrete Prisms." LABEST, Faculty of Engineering of the University of Porto, 2009. 
TACE. "Construção Da Travessia Do Tejo No Carregado Sublanço A1/Benavente, Da A10 Auto-Estrada Buce-Las/Carregado/Ic3: Plano De Qualidade." 2007.

Van der Auweraer, Herman, and Bart Peeters. "International Research Projects on Structural Health Monitoring: An Overview." Structural Health Monitoring 2, no. 4 (2003): 341-58. 


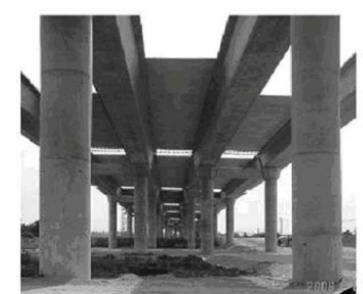

a) general view

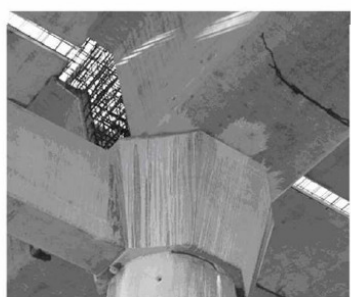

b) diaphragm construction detail

Figure 1. Precast viaduct.

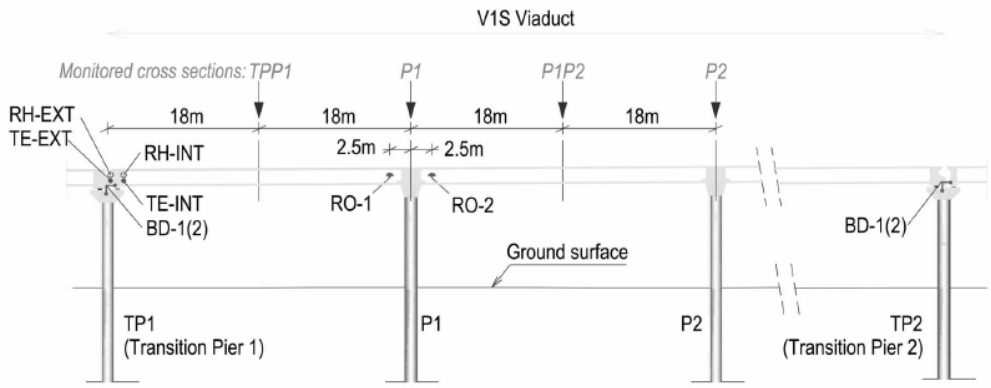

V14S Viaduct

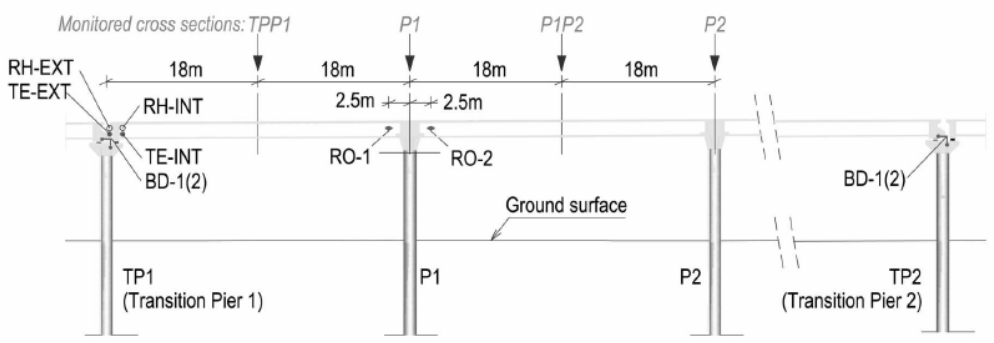

Figure 2. Elevation of the monitored viaducts V1S and V14S.
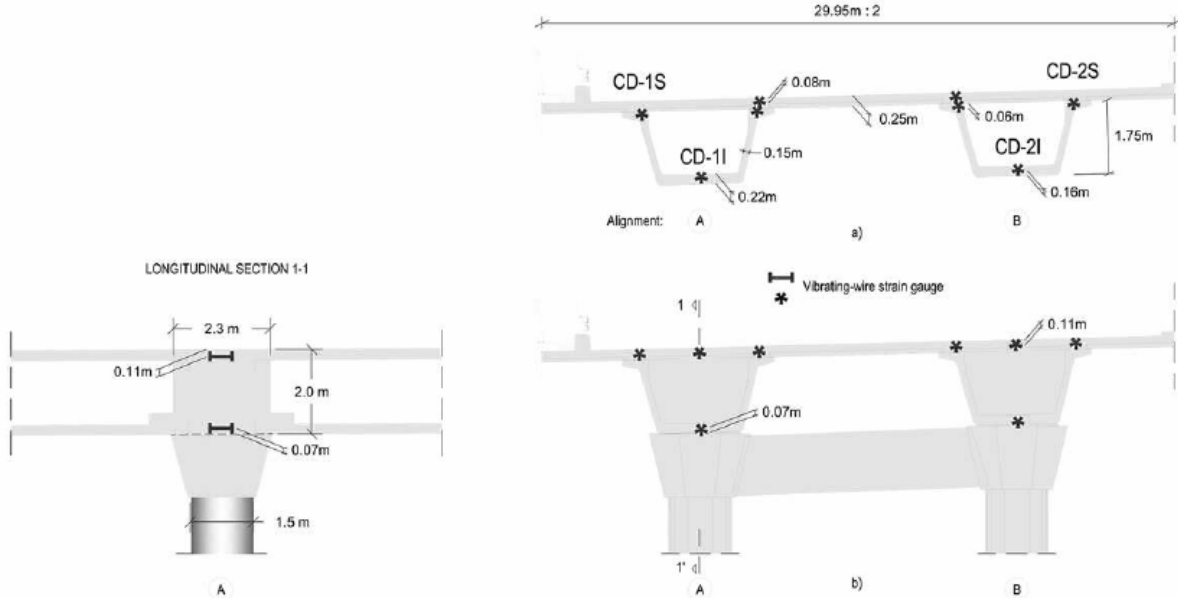

Figure 3. Strain gauges location in the monitored cross sections (a) mid-span cross section, (b) support cross section. 


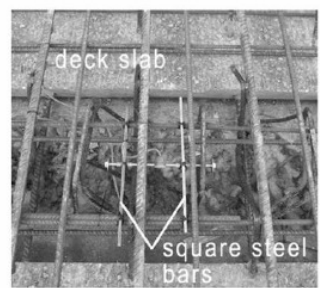

a)

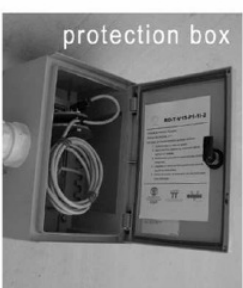

b)

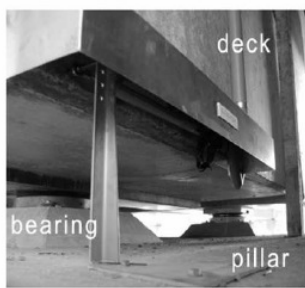

c)

Figure 4. Sensors installation: (a) strain gauge, (b) inclinometer and protection box, (c) displacement transducer and stainless steel shelter.

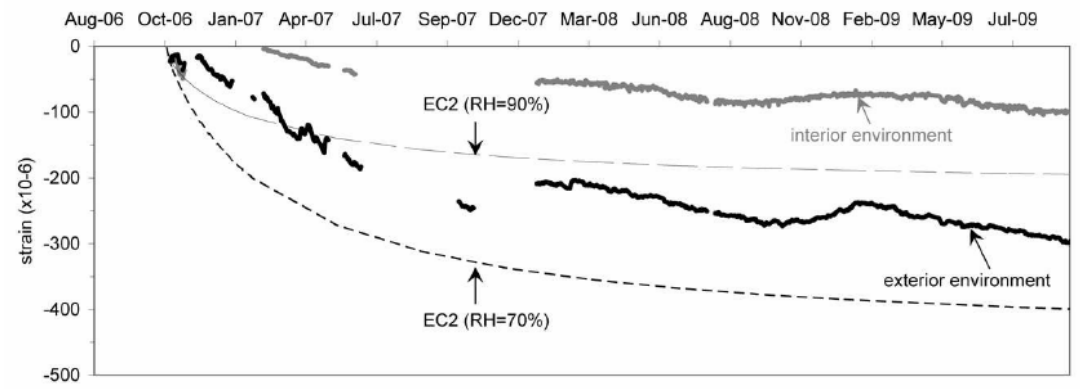

Figure 5. Shrinkage strain of the slab concrete.

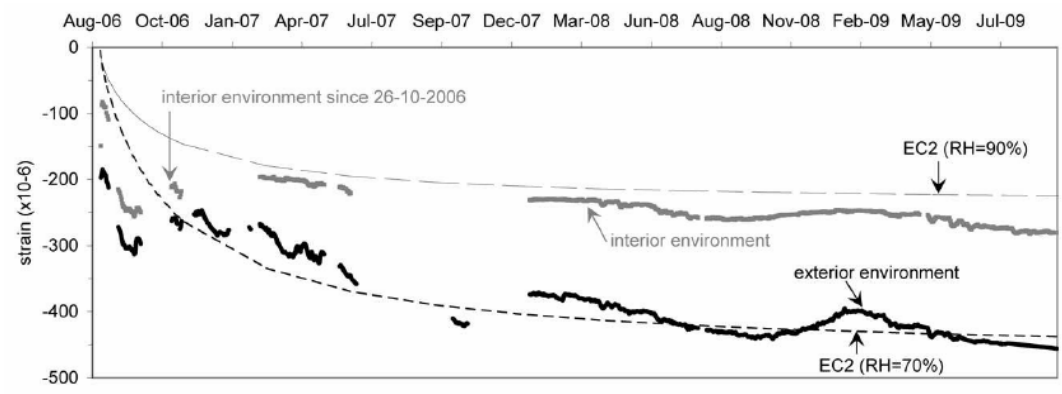

Figure 6. Shrinkage strain of the girder concrete.
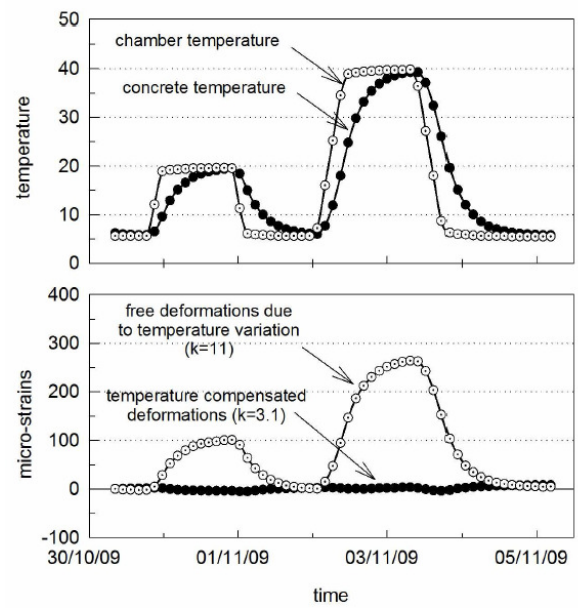

Figure 7. Chamber climatic test. 

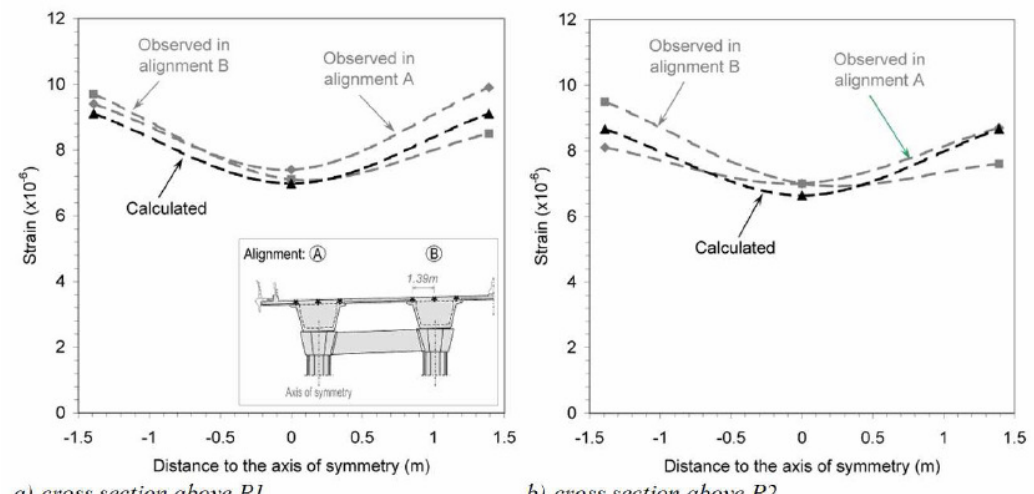

a) cross section above PI

b) cross section above $P 2$

Figure 8. Deck slab strains at the support cross sections during the load test.

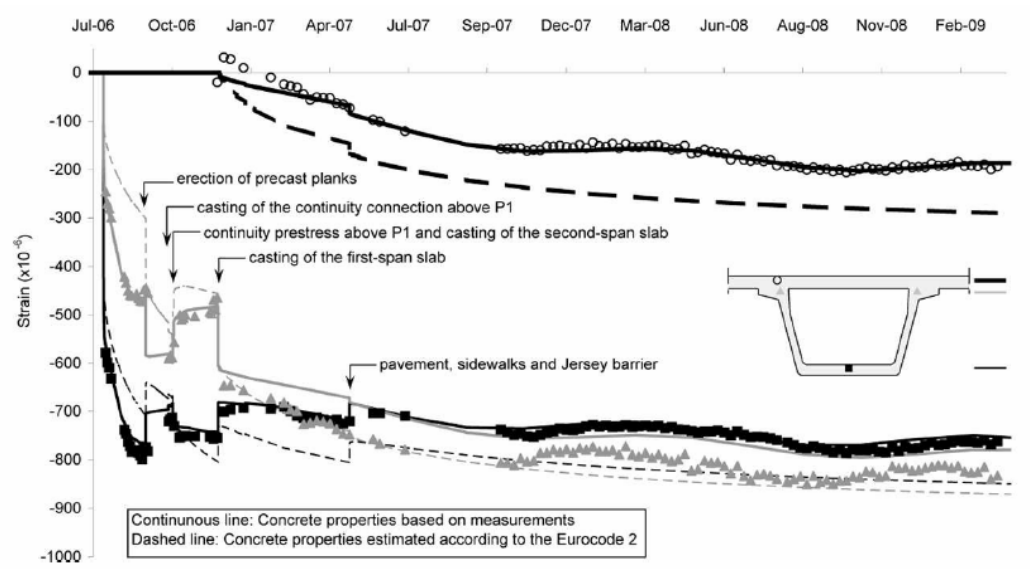

Figure 9. Comparison between numerical and experimental results (strains in the mid-span cross section of the first span, alignment B).
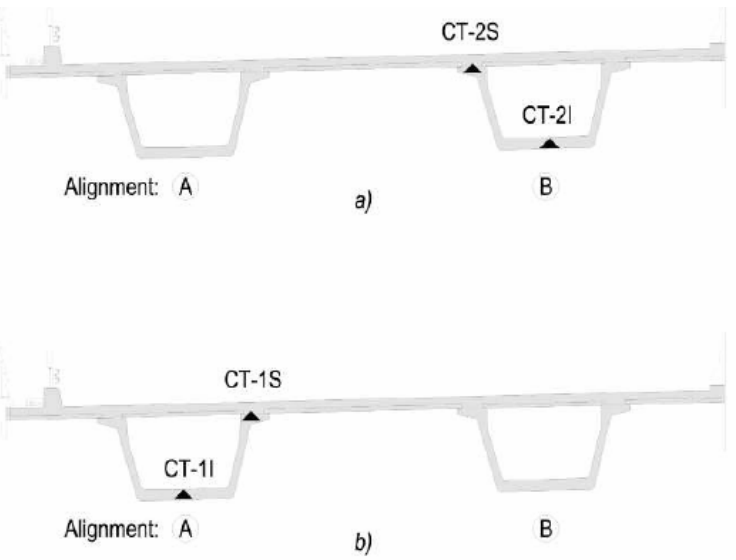

- Embedded temperature sensors

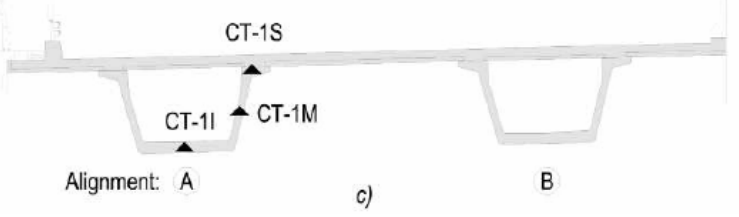

Figure 10. Embedded temperature sensor locations: (a) V1S-TPP1, (b) V1S-P1P2, (c) V14S-P1P2. 

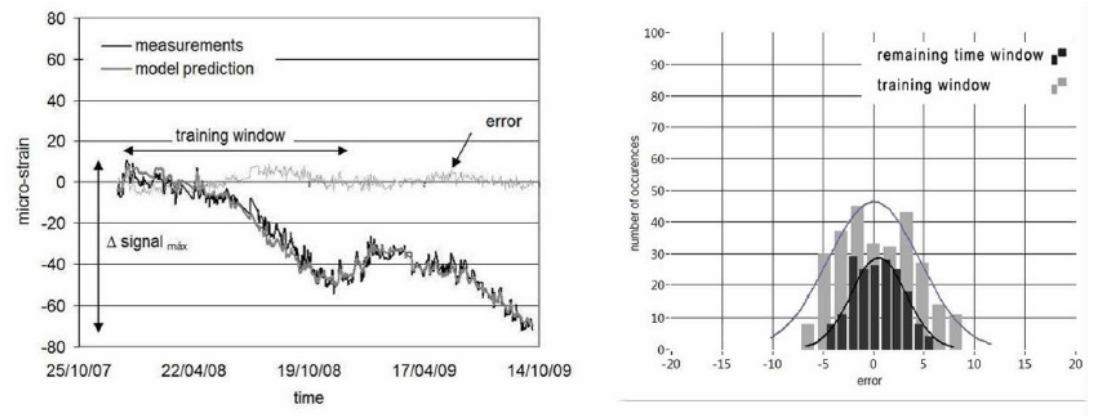

Figure 11. Concrete deformation at the bottom slab of the cross section TPP1 (V1S).
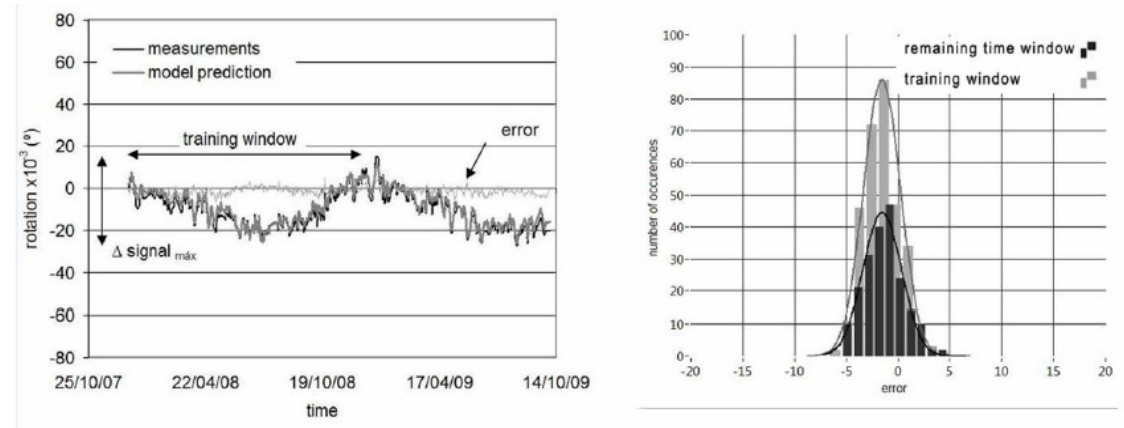

Figure 12. Deck rotation RO-2 of viaduct V14S (see Figure 2).
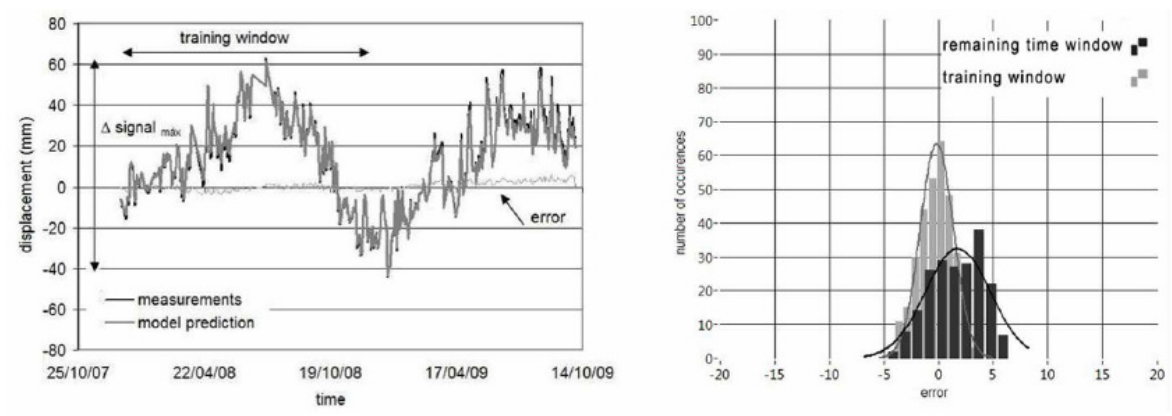

Figure 13. Bearing displacement at the transition pier TP14 of viaduct V14S. 


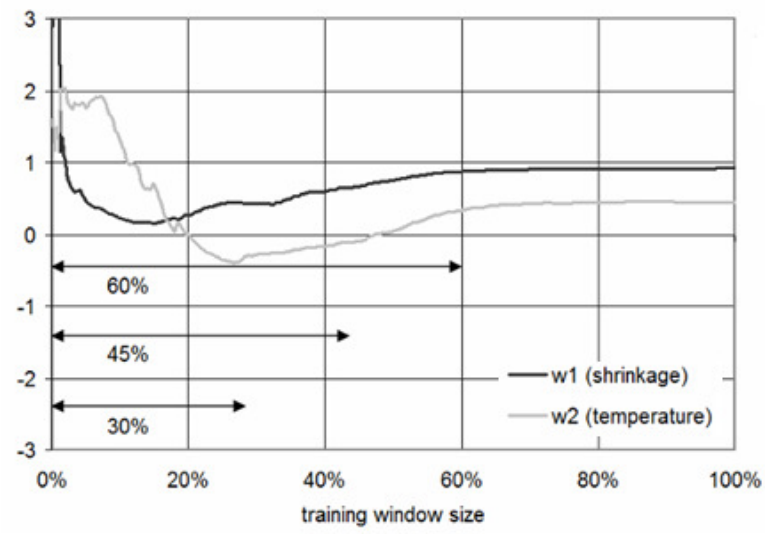

a)

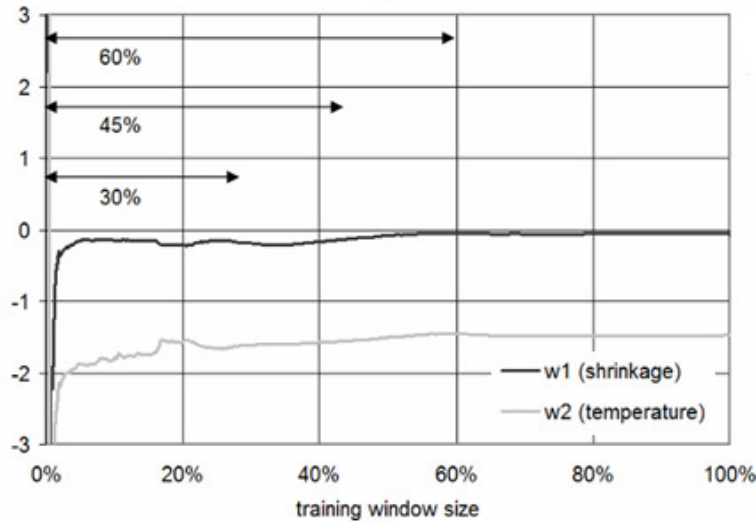

b)

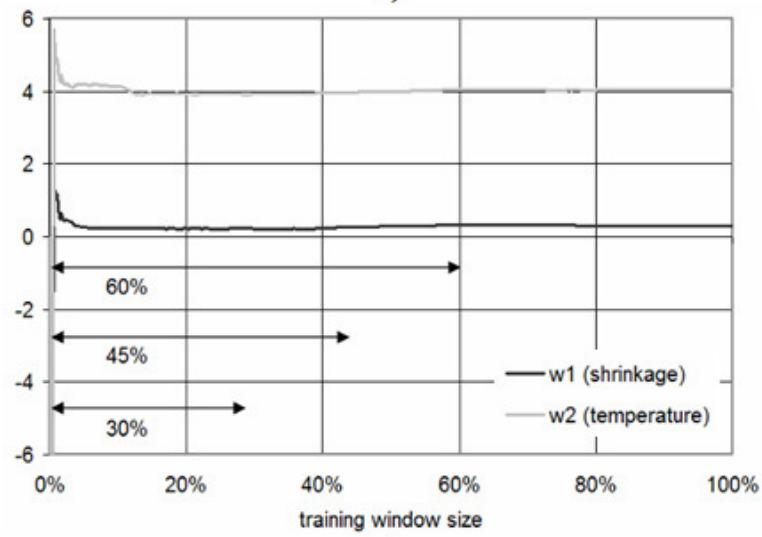

c)

Figure 14. Calculated weights wi as a function of the training window size: (a) concrete deformation at the bottom slab of TPP1(V1S), (b) deck rotation RO-2 (V14S), (c) bearing displacement at TP14 (V14S).

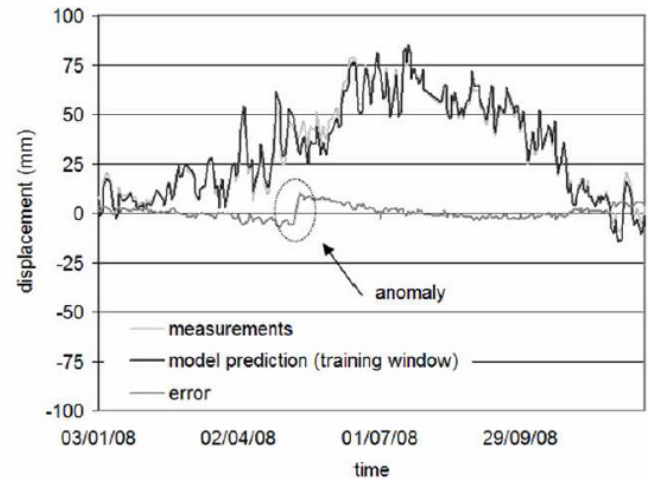

Figure 15. Anomaly detection in bearing displacement BD-TP15 (V14S). 

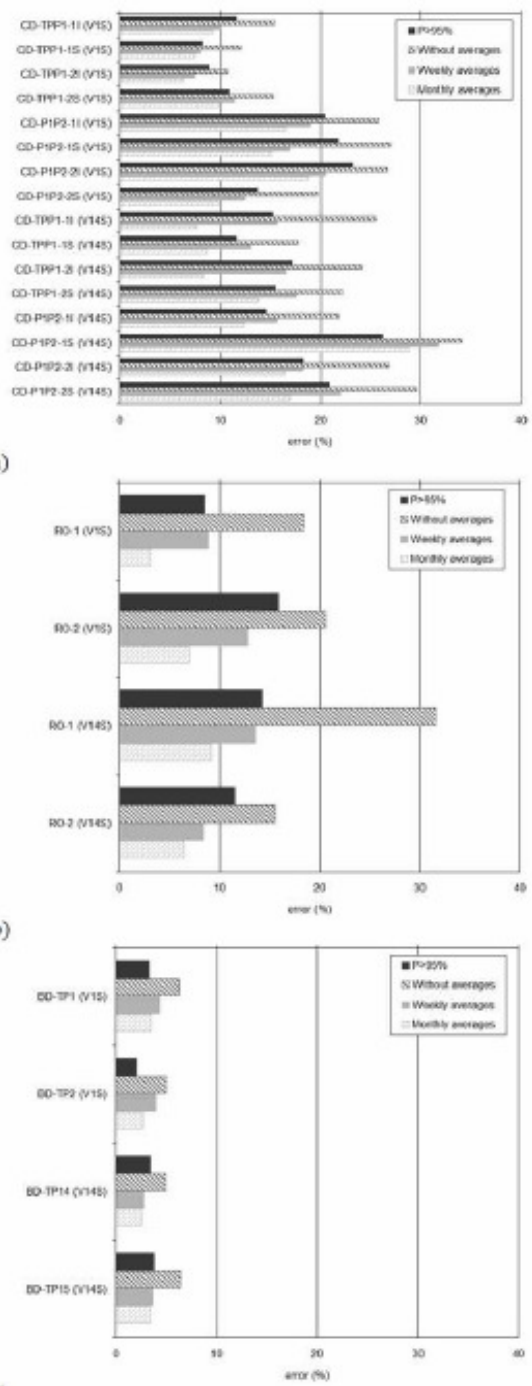

Figure 16. Differences between actual measurements and predictions: (a) concrete deformations, (b) deck rotations, (c) bearing displacements. 
Table 1. Correlation coefficient R2 for the independent measurements in V1S.

\begin{tabular}{|c|cccc|cccc|}
\cline { 2 - 9 } \multicolumn{1}{c|}{} & $\mathrm{SD}_{\mathrm{IN} 1}$ & $\mathrm{SD}_{\mathrm{IN} 2}$ & $\mathrm{SD}_{\mathrm{EX} 1}$ & $\mathrm{SD}_{\mathrm{EX2} 2}$ & $\mathrm{CT}_{11}$ & $\mathrm{CT}_{1 \mathrm{~s}}$ & $\mathrm{CT}_{21}$ & $\mathrm{CT}_{2 \mathrm{~S}}$ \\
\hline $\mathrm{SD}_{\mathrm{IN} 1}$ & 1 & 0.997 & 0.970 & 0.977 & -0.232 & -0.212 & -0.234 & -0.210 \\
$\mathrm{SD}_{\mathrm{N} 2}$ & & 1 & 0.980 & 0.979 & -0.288 & -0.269 & -0.290 & -0.267 \\
$\mathrm{SD}_{\mathrm{EX} 1}$ & & & 1 & 0.983 & -0.414 & -0.393 & -0.415 & -0.391 \\
$\mathrm{SD}_{\mathrm{EX} 2}$ & & & & 1 & -0.259 & -0.238 & -0.260 & -0.236 \\
\hline $\mathrm{CT}_{11}$ & & & & & 1 & 0.995 & 0.990 & 0.994 \\
$\mathrm{CT}_{15}$ & & & & & & 1 & 0.995 & 0.999 \\
$\mathrm{CT}_{21}$ & & & & & & & 1 & 0.994 \\
$\mathrm{CT}_{2 \mathrm{SS}}$ & & & & & & & & 1 \\
\hline
\end{tabular}

Table 2. Correlation coefficient R2 for the independent measurements in V14S.

\begin{tabular}{|c|cccc|ccc|}
\cline { 2 - 8 } \multicolumn{1}{c|}{} & $\mathrm{SD}_{\mathrm{IN} 1}$ & $\mathrm{SD}_{\mathrm{IN} 2}$ & $\mathrm{SD}_{\mathrm{EX1}}$ & $\mathrm{SD}_{\mathrm{EX} 2}$ & $\mathrm{CT}_{1 \mathrm{I}}$ & $\mathrm{CT}_{1 \mathrm{M}}$ & $\mathrm{CT}_{15}$ \\
\hline $\mathrm{SD}_{\mathrm{NN} 1}$ & 1 & 0.962 & 0.980 & 0.966 & -0.573 & -0.572 & -0.556 \\
$\mathrm{SD}_{\mathrm{IN} 2}$ & & 1 & 0.953 & 0.991 & -0.628 & -0.628 & -0.612 \\
$\mathrm{SD}_{\mathrm{EX} 1}$ & & & 1 & 0.958 & -0.439 & -0.438 & -0.422 \\
$\mathrm{SD}_{\mathrm{EX} 2}$ & & & & 1 & -0.595 & -0.593 & -0.580 \\
\hline $\mathrm{CT}_{11}$ & & & & & 1 & 0.999 & 0.995 \\
$\mathrm{CT}_{1 \mathrm{M}}$ & & & & & & 1 & 0.995 \\
$\mathrm{CT}_{1 \mathrm{~S}}$ & & & & & & & 1 \\
\hline
\end{tabular}

Table 3. Maximum relative error for the concrete deformation at the bottom slab of TPP1 (V1S).

\begin{tabular}{cccc}
\hline Maximum & \multicolumn{3}{c}{ Training window size (percentage of the observation window) } \\
\cline { 2 - 4 } relative error & $30 \%$ & $45 \%$ & $60 \%$ \\
\hline Without averages & $39.9 \%$ & $20.2 \%$ & $10.8 \%$ \\
Weekly average & $36.3 \%$ & $16.1 \%$ & $7.5 \%$ \\
Monthly average & $26.9 \%$ & $12.8 \%$ & $6.5 \%$ \\
P $>95 \%$ & $47.2 \%$ & $19.1 \%$ & $8.6 \%$ \\
\hline
\end{tabular}

Table 4. Maximum relative error for the deck rotation RO-2 (V14S).

\begin{tabular}{cccc}
\hline Maximum & \multicolumn{3}{c}{ Training window size (percentage of the observation window) } \\
\cline { 2 - 4 } relative error & $30 \%$ & $45 \%$ & $60 \%$ \\
\hline Without averages & $26.5 \%$ & $17.9 \%$ & $15.6 \%$ \\
Weekly average & $22.8 \%$ & $14.8 \%$ & $8.4 \%$ \\
Monthly average & $20.2 \%$ & $12.5 \%$ & $6.4 \%$ \\
P $>95 \%$ & $23.9 \%$ & $14.7 \%$ & $11.5 \%$ \\
\hline
\end{tabular}

Table 5. Maximum relative error for the bearing displacement at TP14 (V14S).

\begin{tabular}{cccc}
\hline Maximum & \multicolumn{4}{c}{ Training window size (percentage of the observation window) } \\
\cline { 2 - 4 } relative error & $30 \%$ & $45 \%$ & $60 \%$ \\
\hline Without averages & $5.4 \%$ & $4.8 \%$ & $5.5 \%$ \\
Weekly average & $5.0 \%$ & $3.2 \%$ & $2.7 \%$ \\
Monthly average & $3.7 \%$ & $2.0 \%$ & $2.1 \%$ \\
P $>95 \%$ & $2.2 \%$ & $3.0 \%$ & $3.1 \%$ \\
\hline
\end{tabular}

